\title{
ACO Awareness and Perceptions Among Specialists Versus Primary Care Physicians: a Survey of a Large Medicare Shared Savings Program
}

$\mathrm{J}$ Gen Intern Med 37(2):492-4

DOI: $10.1007 /$ s11606-020-06556-w

(C) Society of General Internal Medicine 2021

\section{BACKGROUND}

Medicare Shared Savings Program (MSSP) Accountable Care Organizations (ACOs) have achieved only modest savings. ${ }^{1,2}$ While ACO leaders and policymakers have focused on engaging primary care physicians (PCPs) to improve care coordination and reduce spending, ${ }^{3}$ less attention has been devoted to the medical and surgical specialists who provide expensive services that drive Medicare spending. Moreover, few surveys have explored awareness and perceptions of physicians participating in ACOs, including the views of specialists. Therefore, we compared ACO awareness and perceptions between PCPs and specialists.

\section{METHODS}

We analyzed data from a survey administered in 2018 to clinicians in the Physician Organization of Michigan ACO, ${ }^{4}$ which at the time was the largest MSSP ACO in Michigan and among the ten largest nationally. The present analysis focused on non-pediatrician physician respondents $(n=1022,34 \%$ response rate) practicing within 10 provider organizations. Our primary exposure was whether the respondent was a PCP versus specialist (internal medicine subspecialist, surgeon, other specialist [e.g., radiologist, neurologist]). Outcomes were whether respondents knew they were in an ACO and whether they perceived that joining an ACO had influenced clinical practice, patient outcomes (e.g., health of complex patients), and professional outcomes (e.g., job satisfaction, administrative burden). We fit separate multivariable fixed-effect models to test within-provider organization associations between physician type and each study outcome, adjusting for respondent gender, age, and professional activity (patient care, teaching, research, administration/management, other). We used multiple imputation and post-stratification survey weights to address missing data and survey non-

Received October 13, 2020

Accepted December 22, 2020

Published online January 26, 2021 response, respectively. We used complete data on respondent organization, gender, and specialty to impute missing data on age (4\%), professional activity (4\%), and ACO awareness and perceptions (interquartile range, $1-4 \%$ ).

\section{RESULTS}

Physician respondents included PCPs (23\%) and specialists (77\%), including internal medicine subspecialists (20\%), surgeons (14\%), and other specialists (43\%). Specialists were less likely to be aware of ACO participation and incentives (Fig. 1). Compared to PCPs, specialists were 25 percentage points ([pp]; 95\% confidence interval [CI], -33 to -17 ) less likely to know that they were in an ACO (43\% vs. $69 \%)$. In addition, specialists were $18 \mathrm{pp}(95 \% \mathrm{CI},-26$ to -10$)$ less likely to know that their ACO was accountable for both spending and quality or that their ACO had lowered spending in the previous year $(-7 \mathrm{pp} ; 95 \% \mathrm{CI},-13$ to -1$)$. Specialists were also less likely to perceive that joining an ACO had changed how they practiced medicine $(-9 \mathrm{pp} ; 95 \% \mathrm{CI},-17$ to -2 ), their compensation ( $-11 \mathrm{pp} ; 95 \% \mathrm{CI},-19$ to -3 ), or whether they received useful performance feedback $(-13 \mathrm{pp}$; $95 \%$ CI, -20 to -6 ).

Specialists were less likely to perceive that the ACO had improved patient or professional outcomes (Fig. 2), including care coordination ( $-19 \mathrm{pp}$; 95\% CI, -26 to -11$)$, management between visits ( $-15 \mathrm{pp} ; 95 \% \mathrm{CI},-22$ to -8 ), medically complex patients' health $(-10 \mathrm{pp} ; 95 \% \mathrm{CI},-17$ to -3$)$, professional satisfaction ( $-8 \mathrm{pp} ; 95 \%,-13$ to -3$)$, finances $(-7 \mathrm{pp} ; 95 \% \mathrm{CI},-12$ to -2$)$, staff morale $(-6 \mathrm{pp} ; 95 \% \mathrm{CI},-$ 10 to -2$)$, or administrative burden ( $-5 \mathrm{pp} ; 95 \% \mathrm{CI},-9$ to 2). In supplemental heterogeneity analyses, ACO awareness and perceptions did not systematically vary across the three specialist subgroups, with significant differences for only 3 of 23 measures (data available upon request).

\section{DISCUSSION}

In a large survey of physicians in a single MSSP ACO, we found that participating specialists demonstrated limited awareness and perceptions regarding the ACO. Our study extends prior surveys, demonstrating that internal medicine subspecialists, surgeons, and other specialists were 


\begin{tabular}{|c|c|c|c|c|}
\hline & $\begin{array}{l}\mathrm{PCP} \\
\text { mean }\end{array}$ & $\begin{array}{l}\text { Specialist } \\
\text { mean }\end{array}$ & $\begin{array}{l}\text { Difference } \\
(95 \% \mathrm{Cl})\end{array}$ & $P$ value \\
\hline \multicolumn{5}{|l|}{ ACO Awareness ( $\%$ know) } \\
\hline Participate in an $\mathrm{ACO}$ & 69 & 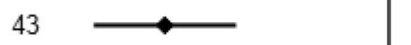 & $-25(-33,-17)$ & $<.001$ \\
\hline Held accountable for both spending and quality. & 47 & 29 & $-18(-26,-10)$ & $<.001$ \\
\hline Lowered spending in the most recent performance year & 30 & 22 & $-7(-13,-1)$ & 0.022 \\
\hline Held accountable for only Medicare patients & 11 & 7 & $-3(-8,1)$ & 0.141 \\
\hline Did not receive a bonus from Medicare in most recent year & 16 & 14 & $-3(-7,1)$ & 0.197 \\
\hline Does not face downside financial risk, i.e., cannot lose money & 8 & 5 & $-3(-6,1)$ & 0.182 \\
\hline \multicolumn{5}{|l|}{ Perceived ACO influence on clinical practice ( $\%$ agree) } \\
\hline I receive useful feedback on $\mathrm{ACO}$ cost and quality performance & 45 & 32 & $-13(-20,-6)$ & 0.001 \\
\hline Joining an ACO has changed how I am compensated & 45 & 34 & $-11(-19,-3)$ & 0.009 \\
\hline Joining an ACO has changed how I practice medicine & 28 & 18 & $-9(-17,-2)$ & 0.011 \\
\hline Joining an $\mathrm{ACO}$ has made me more aware of controlling costs & 50 & 46 & $-4(-11,4)$ & 0.358 \\
\hline ACO financial bonuses are large enough to influence my behavior & 26 & 25 & $-0(-6,5)$ & 0.907 \\
\hline & & 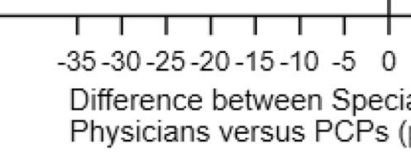 & & \\
\hline
\end{tabular}

Figure 1 ACO awareness and perceived ACO influence on clinical practice. Note: ACO denotes accountable care organization. PCP denotes primary care physician. CI denotes confidence interval. PP denotes percentage point. Models are described in the "METHODS" section. Survey weights were applied to generalize to the Physician Organization of Michigan ACO. Multiple imputation was used for missing data. Estimated differences may differ from differences between PCP and specialist means due to rounding. For ACO awareness, 1, correct; 0, incorrect or do not know. For perceived ACO influence on clinical practice, 1 , moderately or strongly agree; 0 , moderately or strongly disagree. PCP $(n=231)$ was defined as General Internal Medicine, Primary Care, Geriatrics, Hospitalist, General Practice, Palliative Medicine, or Preventive Medicine. Specialist $(n=791)$ was defined as Internal Medicine Subspecialist $(n=185)$, Surgeon $(n=147)$, or Other Physician Specialist $(n=459)$ such as Radiology, Anesthesiology, and Emergency Medicine.

substantially less likely than PCPs to be aware of ACO participation or to perceive that joining an ACO had changed clinical practice or improved patient or professional outcomes. ${ }^{4,5}$ This lack of engagement across specialists may also

$$
\begin{aligned}
& \text { PCP Specialist } \\
& \text { mean mean } \\
& \text { Difference } \\
& (95 \% \mathrm{Cl}) \quad \text { P value }
\end{aligned}
$$

Perceived ACO effect on patient outcomes (\% positive)

Coordinate care across care settings

Help patients manage care between visits

Improve health of medically complex patients

Engage in shared decision-making

Improve health of low-income patients

Reduce inappropriate or harmful care

Decrease unnecessary hospitalizations

$\begin{array}{ll}39 & 20 \\ 37 & 22 \\ 26 & 16 \\ 22 & 16 \\ 17 & 14 \\ 17 & 14 \\ 22 & 21\end{array}$
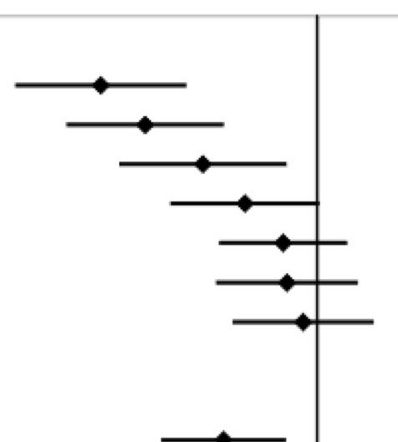

\footnotetext{
Perceived ACO effect on professional outcomes (\% positive)

Your professional satisfaction

The financial situation of your practice or hospital as a whole

Staff morale

The administrative burden on your practice or hospital

Your autonomy as a clinician
}

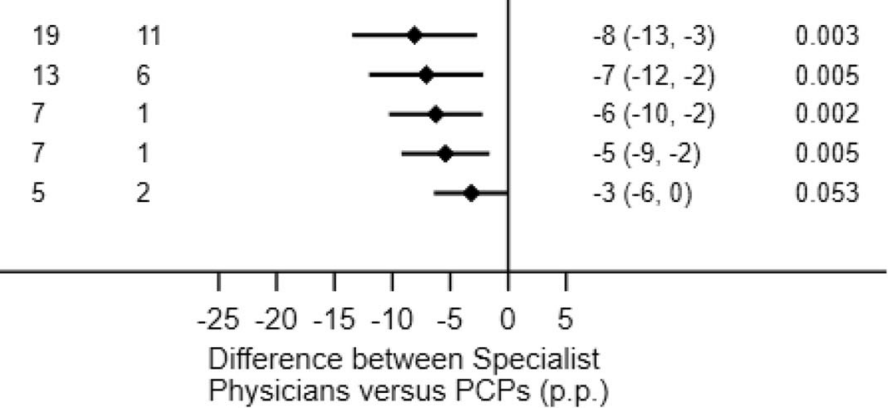

Figure 2 Perceived ACO effect on patient and professional outcomes. Note: ACO denotes accountable care organization. PCP denotes primary care physician. CI denotes confidence interval. PP denotes percentage point. Models are described in the main text. Survey weights were applied to generalize to the Physician Organization of Michigan ACO. Multiple imputation was used for missing data. Estimated differences may differ from differences between PCP and specialist means due to rounding. For perceived ACO effect on patient and professional outcomes, 1, positive impact; 0 , no impact, negative impact, or do not know. 
help to explain ACOs' modest impact on spending and quality. ${ }^{6}$ The generalizability of our findings is limited by data from a single large MSSP ACO. Response bias is possible given the moderate response rate, though this appears unlikely given respondents' limited awareness or opinions. These limitations notwithstanding, our study suggests a pressing need for ACOs to engage both PCPs and specialists in care redesign. Further research is needed to determine the most effective strategies for accomplishing this.

Acknowledgments: This work benefited from guidance on survey development and administration from Lisa Holland, MA; Tedi Engler, $B S$; and Eve Kerr, MD, MPH (University of Michigan), without compensation. The authors acknowledge the Physician Organization of Michigan ACO for its commitment to evaluation and research.

Adam A. Markovitz, $P h D^{1}$

Andrew M. Ryan, PhD, $M A^{2}$

Timothy A. Peterson, $M D, M B A^{3}$

Michael D. Rozier, $\mathrm{PhD}^{4}$

John Z. Ayanian, MD, MPP

John M. Hollingsworth, $M D, M S^{6}$

${ }^{1}$ University of Michigan Medical School,

Ann Arbor, MI, USA

${ }^{2}$ Department of Health Management and Policy,

University of Michigan School of Public Health,

Ann Arbor, MI, USA

${ }^{3}$ Department of Emergency Medicine, University of Michigan Medical School,

Ann Arbor, MI, USA

${ }^{4}$ Department of Health Management and Policy, Saint Louis University,

St. Louis, MO, USA

${ }^{5}$ Institute for Healthcare Policy and Innovation,

University of Michigan,

Ann Arbor, MI, USA

${ }^{6}$ Dow Division of Health Services Research, Department of Urology, University of Michigan

Medical School,

Ann Arbor, MI, USA

Corresponding Author: John M. Hollingsworth, MD, MS; Dow Division of Health Services Research, Department of Urology, University of Michigan Medical School, Ann Arbor, MI, USA (e-mail:kinks@med.umich.edu).
Funding Dr. Hollingsworth was supported by National Institute on Aging grant R01AG068074. Dr. Markovitz was supported by the Horowitz Foundation for Social Policy, Agency for Healthcare Research and Quality grant R36HSO25615, and the University of Michigan Rackham Hammel Research Award. Dr. Ryan was supported by National Institute on Aging grant R01AG047932. Dr. Ayanian was supported by National Institute on Aging grant 3P01AG032952. The Physician Organization of Michigan ACO provided funds for survey mailings.

\section{Compliance with Ethical Standards:}

Conflict of Interest: Dr. Peterson serves as ACO Executive for the Physician Organization of Michigan ACO and receives partial salary support for this position. Dr. Norton and Dr. Rozier have no relevant disclosures. The remaining author disclosures are listed in the "Funding" statement.

Role of the Funder/Sponsor: The funding organizations had no role in the design and conduct of the study; collection, management, anal$y$ sis, and interpretation of the data; preparation, review, or approval of the manuscript; and decision to submit the manuscript for publication.

\section{REFERENCES}

1. Markovitz AA, Hollingsworth JM, Ayanian JZ, Norton EC, Yan PL, Ryan AM. Performance in the Medicare Shared Savings Program after accounting for nonrandom exit: an instrumental variable analysis. Ann Intern Med. 2019; 171(1):27-36. https://doi.org/10.7326/M18-2539

2. McWilliams JM, Hatfield LA, Landon BE, Hamed P, Chernew ME. Medicare spending after 3 years of the Medicare Shared Savings Program. N Engl J Med. 2018; 379(12):1139-1149. https://doi.org/10.1056/ NEJMsa1803388

3. Lewis VA, Schoenherr $\mathbf{K}$, Fraze T, Cunningham A. Clinical coordination in accountable care organizations: A qualitative study. Health Care Manage Rev. Published online 2019. https://doi.org/10.1097/HMR. 0000000000000141

4. Markovitz AA, Rozier MD, Ryan AM, et al. Low-value care and clinician engagement in a marge Medicare Shared Savings Program ACO: a survey of frontline clinicians. J Gen Intern Med. 2020; 35(1): 133-141. https://doi. org/10.1007/s11606-019-05511-8

5. Schur CL, Sutton JP. Physicians in Medicare ACOs offer mixed views of model for health care cost and quality. Health Aff. 2017; 36(4):649-654. https://doi.org/10.1377/hlthaff.2016.1427

6. Sukul D, Ryan AM, Yan P, et al. Cardiologist participation in accountable care organizations and changes in spending and quality for Medicare patients with cardiovascular disease. Circ Cardiovasc Qual Outcomes. Published online 2019. https://doi.org/10.1161/CIRCOUTCOMES.118. 005438

Publisher's Note Springer Nature remains neutral with regard to jurisdictional claims in published maps and institutional affiliations. 